\title{
Dyslexic and nondyslexic reading fluency: Rapid automatized naming and the importance of continuous lists
}

\author{
Manon W. Jones, Holly P. Branigan, ANd M. Louise Kelly \\ University of Edinburgh, Edinburgh, Scotland
}

\begin{abstract}
Rapid automatized naming (RAN; Denckla \& Rudel, 1976) tasks are consistent predictors of fluency that also discriminate between dyslexic and nondyslexic reading groups. The component processes of RAN that are responsible for its relationship with reading ability remain underspecified, however. We report a study on dyslexic and nondyslexic adult groups that experimentally manipulated RAN formats to elucidate how different components of RAN differentially influence dyslexic and nondyslexic performance. The dyslexic group showed a pervasive deficit in rapid access of individually presented items. Additionally, they showed a significant impairment when multiple items were presented, whereas nondyslexic readers showed marginal facilitation for this format. We discuss the implications of these findings with respect to reading-group differences in reading fluency.
\end{abstract}

An established core deficit of developmental dyslexia is difficulty in manipulating phonemes within words (see Snowling, 2000); however, Wolf and Bowers (1999) proposed an additional core fluency deficit that is independent of phonological impairment. Rapid automatized naming (RAN; Denckla \& Rudel, 1976) tasks are a well-known independent predictor of reading fluency (see, e.g., Lervåg \& Hulme, in press; Manis, Doi, \& Bhadha, 2000; Young $\&$ Bowers, 1995). RAN involves visually presented arrays of high-frequency items (letters, digits, colors, or objects) that are repeated multiple times in a randomized order, typically five items, each repeated 10 times across five rows. The participant names all the stimuli, from left to right across the page, as quickly as possible.

RAN performance consistently discriminates dyslexic from nondyslexic readers (see, e.g., Denckla \& Rudel, 1976; see Wolf \& Bowers, 1999, for a review). In orthographically shallow languages, it also contributes more variance than phonological decoding to reading ability (Wimmer, Mayringer, \& Landerl, 2000). Naming rates for alphanumeric stimuli in particular remain strong predictors of reading ability (Wolf \& Obregón, 1992) that persist into adulthood (Shaywitz \& Shaywitz, 2005). As a measure of reading fluency, therefore, RAN has strong potential to explain differences in reading ability, and at least 104 studies published since 1990 have used RAN as a measure of reading skill. ${ }^{1}$ Despite this body of research, the underlying component processes that determine RAN's relationship with reading ability remain poorly specified (Georgiou, Parrila, \& Kirby, 2006).

The present article reports a study that isolated component processes of RAN to elucidate which aspects of the task discriminate between dyslexic and nondyslexic readers' naming speeds. By extension, we examined the low-level (graphemic and phonological) processing that is involved in reading fluency.

\section{Components of RAN}

Performance on RAN is often assumed to reflect "retrieval of phonological codes from a long-term store" (Wagner, Torgesen, Laughon, Simmons, \& Rashotte, 1993, p. 84), and impaired RAN performance is often assumed to reflect phonological deficits (Clarke, Hulme, \& Snowling, 2005). A number of studies have also shown significant shared variance between RAN and phonologicalawareness constructs (e.g., Savage et al., 2005; Torgesen, Wagner, Rashotte, Burgess, \& Hecht, 1997). Savage et al., for example, found that RAN, phonological awareness, and motor balance loaded onto one component.

Other evidence, however, suggests that RAN makes a unique contribution to reading fluency when phonological skill is factored out (see, e.g., Powell, Stainthorp, Stuart, Garwood, \& Quinlan, 2007). Research has also suggested that multi-item processing is key to understanding RAN's relationship with reading fluency. Studies have demonstrated a stronger relationship between reading fluency and continuous (multiple, matrix presentation of items) versions of RAN than that between reading fluency and discrete (individual letter presentation) versions (Bowers \& Swanson, 1991; Walsh, Price, \& Gillingham, 1988). The difference between continuous and discrete versions is a matter of complexity: Continuous versions implicate not only access to the graphemic and phonological properties of stimuli, but also other processes, such as saccadic

M.W. Jones, manon.wyn.jones@ed.ac.uk 
eye movements and sequencing of multiple items, which in itself requires inhibition of previous (already named) stimuli and efficient processing of upcoming items. In contrast, naming discrete items measures only graphemic and phonological access of individual items, because it removes oculomotor and sequencing requirements from the task. There is some evidence that both discrete and continuous lists discriminate dyslexic from nondyslexic readers (Castel, Pech-Georgel, George, \& Ziegler, 2008). The stronger relationship of continuous lists to reading fluency suggests, however, that continuous lists may be particularly difficult for dyslexic readers.

In the present study, we examined the importance of additional oculomotor and sequencing task requirements to RAN performance across nondyslexic and dyslexic groups. Both aspects might be relevant to disparities in group performance. First, there is some evidence that oculomotor control may be impaired in dyslexia (Biscaldi, Gezeck, \& Stuhr, 1998), although this finding is not robust (Hutzler, Kronbichler, Jacobs, \& Wimmer, 2006).

Second, recent evidence suggests that dyslexic readers find it more difficult than do nondyslexic readers to sequence multiple items, in terms of both inhibiting previously activated information and processing upcoming items (Jones, Obregón, Kelly, \& Branigan, 2008). Studies investigating the effect of multi-item presentation on nondyslexic readers' task performance have found that flanking items (typically, graphemes or arbitrary symbols) interfere with discrimination of a target item (e.g., Bouma, 1971). "Crowding effects" are particularly strong in dyslexic readers, who show higher error rates for flanked targets than do nondyslexic readers (see, e.g., Pernet, Valdois, Celsis, \& Démonet, 2006). The deficit in dyslexic groups has been attributed to impaired "perceptual noise exclusion" (Sperling, Lu, Manis, \& Seidenberg, 2005), but recent evidence suggests that multi-item processing impairs the connection between a visual stimulus and its phonological counterpart (Hawelka \& Wimmer, 2005, 2008; Jones et al., 2008).

For nondyslexic readers, the effect of multi-item presentation on identification of a target varies as a function of the items themselves and the task requirements. Crowding effects are reduced, for example, when the target and distractor items are dissimilar (e.g., Kooi, Toet, Tripathy, \& Levi, 1994), and it is likely that top-down influences contribute to the extent of a crowding effect (Levi, 2008). When naming sequences of pictures, nondyslexic participants activate extrafoveal phonological features of upcoming items (in position $n+1$ ) in the array, which facilitates processing times (Morgan \& Meyer, 2005). Studies of text reading have also shown that for nondyslexic readers, the availability of upcoming words in the parafovea (an area up to $2^{\circ}$ of visual angle from the fovea) can increase the speed with which text is read. Parafoveal processing of upcoming words largely determines decisions about where to make the next fixation and whether the next word requires longer processing time (Sereno \& Rayner, 2000); hence, nondyslexic readers show preview benefits during text reading.
In contrast, dyslexic readers do not show preview benefits for lexical items (Chace, Rayner, \& Well, 2005; Jones, Kelly, \& Corley, 2007). Chace et al. suggested that dyslexic readers' increased processing difficulty in foveal regions precludes allocation of attention to parafoveal regions. Jones et al. (2008) showed, however, that dyslexic readers are sensitive to parafoveal information in RAN, and that parafoveal information is in fact a potential source of confusion. These findings suggest a potential dissociation in RAN performance - and by extension, reading fluency whereby multi-item processing facilitates nondyslexic naming times but impairs dyslexic naming times.

The present study examined the extent to which multiitem processing versus discrete processing in RAN discriminates between nondyslexic and dyslexic readers' naming times. We took the novel step of directly comparing reaction times (RTs) across three RAN variations that represented different levels of complexity: (1) a discretestatic format, in which individual letters were presented serially in a single location (see, e.g., Bowers \& Swanson, 1991 - it required only graphemic and phonological access and, hence, was treated as the baseline condition); (2) a continuous-matrix version (the original and most complex form of RAN); and (3) a novel discrete-matrix version, in which individual letters were presented serially but in positions analogous to the continuous-matrix version (involving saccadic eye movements but no parafoveal preview). The novel discrete-matrix condition measured saccadic efficiency independently of how well parafoveal information was processed (see Table 1). We chose adult groups because adult dyslexics display less variability in naming times (Wolf \& Bowers, 1999), and there is less probability that dyslexia will be confounded with other developmental disabilities, such as attention deficit hyperactivity disorder (Taroyan, Nicolson, \& Fawcett, 2007). We used the RAN letters task because of its enduring relationship with reading ability (Wolf \& Obregón, 1992).

For nondyslexic readers, we predicted that presentation of multiple items would facilitate naming times, since it would allow simultaneous processing of more than one item. We thus predicted that nondyslexic readers would be significantly faster in the continuous-matrix condition than in the (baseline) discrete-static condition. More importantly, group differences between the nondyslexic and dyslexic groups would be informative about the nature of the RAN deficit in dyslexia: If it involves a fundamental impairment in grapheme and/or phonological retrieval, then dyslexic readers should be significantly slower than nondyslexic readers in the discrete-static condition. Moreover, if the deficit additionally involves impaired oculomo-

Table 1

Processes Involved in the Variant RAN Formats

\begin{tabular}{lccc}
\hline \multicolumn{1}{c}{ Process } & $\begin{array}{c}\text { Continuous- } \\
\text { Matrix }\end{array}$ & $\begin{array}{c}\text { Discrete- } \\
\text { Matrix }\end{array}$ & $\begin{array}{c}\text { Discrete- } \\
\text { Static }\end{array}$ \\
\hline $\begin{array}{l}\text { Grapheme/phonological access } \\
\text { Oculomotor control }\end{array}$ & $\bullet$ & $\bullet$ & $\bullet$ \\
Sequencing & $\bullet$ & $\bullet$ & \\
\hline
\end{tabular}

Note-Each bullet signifies a specific processing requirement. 
tor control of eye movements, then dyslexic readers should be slower in the discrete-matrix condition as compared with their own and nondyslexic readers' performance in the discrete-static condition, and with nondyslexic readers' performance in the discrete-matrix condition. Finally, if impairments in multi-item processing also contribute to the RAN deficit, dyslexic readers should be slower in the continuous-matrix condition as compared with their own and nondyslexic readers' performance in the discrete-static condition, and with nondyslexic readers' performance in the continuous-matrix condition.

\section{METHOD}

\section{Participants}

One group of 21 (10 male, 11 female) dyslexic students (mean age $=22.75)$, and one group of 21 (10 male, 11 female) nondyslexic students (mean age $=20.75$ ), all native English speakers, participated for payment. Participants in the dyslexic group were formally diagnosed with dyslexia by an educational psychologist, and they reported no other known linguistic or behavioral disorder. We determined a literacy profile for each participant, comprising a spelling and word-recognition test (Wide Range Achievement Test [WRAT-3]; Wilkinson, 1993), and a nonword reading test that assessed phonological decoding.

\section{Materials and Design}

The experiment comprised three variant presentation methods of the standard RAN test, using the letters $a, s, d, p$, and $o$ (Figure 1) from the original RAN (Denckla \& Rudel, 1976).

Continuous-matrix condition. All letters were simultaneously visible in a continuous $10 \times 5$ matrix (per trial), in accordance with the standard RAN procedure.

Discrete-matrix condition. Individual letters were presented serially in matrix format.

Discrete-static condition. Individual letters were presented serially in a single, centered position.

\section{Procedure}

Letters were presented in Arial 18-point font in black type on a white backgound and at a viewing distance of $1^{\circ}$ on a 15 -in. RM monitor. In the two discrete conditions, a voice-activated relay removed the current letter and triggered presentation of the next letter. The last stimulus of each trial (50th letter) had inverse colors to signify that a keyboard response was required to end the trial. In the continuous-matrix condition, horizontal distance between any two letters subtended an angle of $2^{\circ}$. Each trial comprised 50 randomized presentations of the 5 letters. Participants were given a practice session, comprising two RAN trials in each condition. The experiment was blocked by condition and was counterbalanced across participants. Each block comprised four trials. The session lasted approximately $20 \mathrm{~min}$.

\section{RESULTS}

Although dyslexic readers scored within the normal range (reflecting their status as high-functioning, compensated dyslexic readers), they obtained reliably lower standardized scores than did nondyslexics on the spelling and word-reading sections of the WRAT-3 (Table 2), ${ }^{2}$ and they made more errors on a nonword reading task (Manis, Seidenberg, Doi, McBride-Chang, \& Petersen, 1996).

We calculated the mean participant RT to complete each whole trial. ${ }^{3}$ Participants' performance was analyzed using linear mixed effects (LME) analysis (Baayen, 2008; Bates, Maechler, \& Dai, 2008) to take into account the heterogeneity of participants' trial completion times (see Figure 2). LME models are similar to ANOVAs in that both indicate the effect of experimental manipulations on the dependent variable. LME analysis is particularly useful for analyzing data from heterogeneous groups (such as individuals with dyslexia), in that it allows a separation of the effects of the experimental manipulations (fixed effects) from differences in the baseline performance by participants and items (random effects) (see Jones et al., 2008, for a more detailed account).

The LME analysis comprised a 2 (group: dyslexic, nondyslexic) $\times 3$ (RAN formats continuous-matrix, discrete-matrix, discrete-static) design. Random-effects variables comprised participant and item intercept variances. We treated the nondyslexic, discrete-static condition as the baseline (or intercept) condition against which the other fixed effects were compared. Note that these analyses are reported with $t$ values for each coefficient and with estimated probabilities that are based on 10,000 Markov chain Monte Carlo (MCMC) samples. MCMCs are recommended because of the difficulty in determining degrees of freedom corresponding to each $t$ value for the model coefficients.

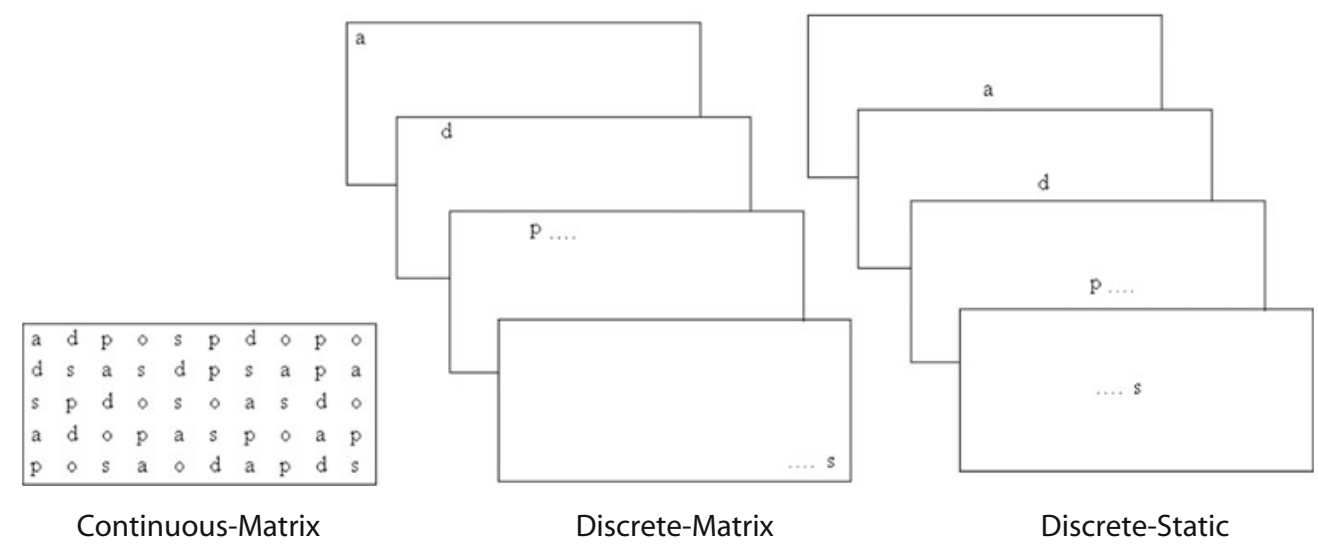

Figure 1. Example frames of a single trial in each condition. 
Table 2

Reading Group Standardized Scores on Measures of Spelling and Reading

Dyslexic Nondyslexic

\begin{tabular}{lrrrrrcc}
\hline & \multicolumn{2}{c}{ Dyslexic } & & \multicolumn{2}{c}{ Nondyslexic } & & \\
\cline { 2 - 3 } & \multicolumn{1}{c}{$M$} & \multicolumn{1}{c}{$S D$} & & \multicolumn{1}{c}{$M$} & $S D$ & $t$ & Cohen's $d$ \\
\hline Spelling & 103.8 & 10.0 & & 116.3 & 7.2 & $4.67^{* *}$ & 1.43 \\
Word reading & 105.5 & 12.6 & & 119.6 & 5.6 & $4.68^{* *}$ & 1.44 \\
Nonword reading & 9.3 & 6.4 & 2.7 & 2.5 & $4.14^{* *}$ & 0.06 \\
\hline
\end{tabular}

Note-Equal variances were not assumed for word reading. Standardized scores were used for spelling and word reading. Nonword-reading scores $=$ errors $/ 44 .{ }^{* *} p<.001$.

The analysis showed that in the nondyslexic group, the discrete-matrix condition was not significantly different from the discrete-static (baseline) condition $(t=.82$, $p=.43)$. The continuous-matrix condition was marginally different from the discrete-static condition $(t=1.71$, $p=.09$ ); however, nondyslexic readers were marginally faster to name multi-item arrays than to name single-item static arrays.

The dyslexic group's performance differed significantly from the nondyslexic group's performance in the discretestatic condition $(t=6.73, p<.0001)$ : Dyslexic readers were significantly slower than nondyslexic readers; however, their performance in the discrete-matrix condition did not differ significantly from the additive contributions to the model of (1) their own performance in the discretestatic condition and (2) nondyslexic readers' performance in the discrete-matrix and discrete-static conditions $(t=$ $.54, p=.64)$. That is, dyslexic readers' performance on the discrete-matrix did not differ from what had already been predicted on the basis of the fixed-effect comparisons that were described above. Crucially, however, dyslexic readers' performance was significantly slower in the continuous-matrix condition when compared with the additive contributions to the model of (1) their own performance in the discrete-static condition and (2) nondyslexic readers' performance in the continuous-matrix and discrete-static conditions $(t=3.21, p<.001)$; hence, dyslexic readers' performance was particularly impaired when multiple items were presented simultaneously.

In sum, dyslexic readers were significantly slower than nondyslexic readers in discrete-static formats - the form of RAN that involves the least complex processing requirements. They were also significantly impaired by multi-item presentation, whereas nondyslexic readers were not. Neither group demonstrated sensitivity to the additional oculomotor requirement that was posed by the discrete-matrix condition relative to the discrete-static condition.

\section{DISCUSSION}

In this study, we examined the underlying processes that determine task performance for nondyslexic and dyslexic groups by directly comparing different presentation formats for RAN tasks.

Dyslexic readers were consistently slower than nondyslexic readers across all presentation formats-even the discrete-static condition, which required no eye movement or sequencing processes. Our study thus extends previous findings of discrete-naming difficulty in dyslexic children (Castel et al., 2008) to a high-functioning adult population. Critically, there were greater discrepancies in performance in the continuous-matrix condition, where multiple items were presented simultaneously. Nondyslexic readers were not slower to name items in the continuous-matrix condition than in the discrete-static

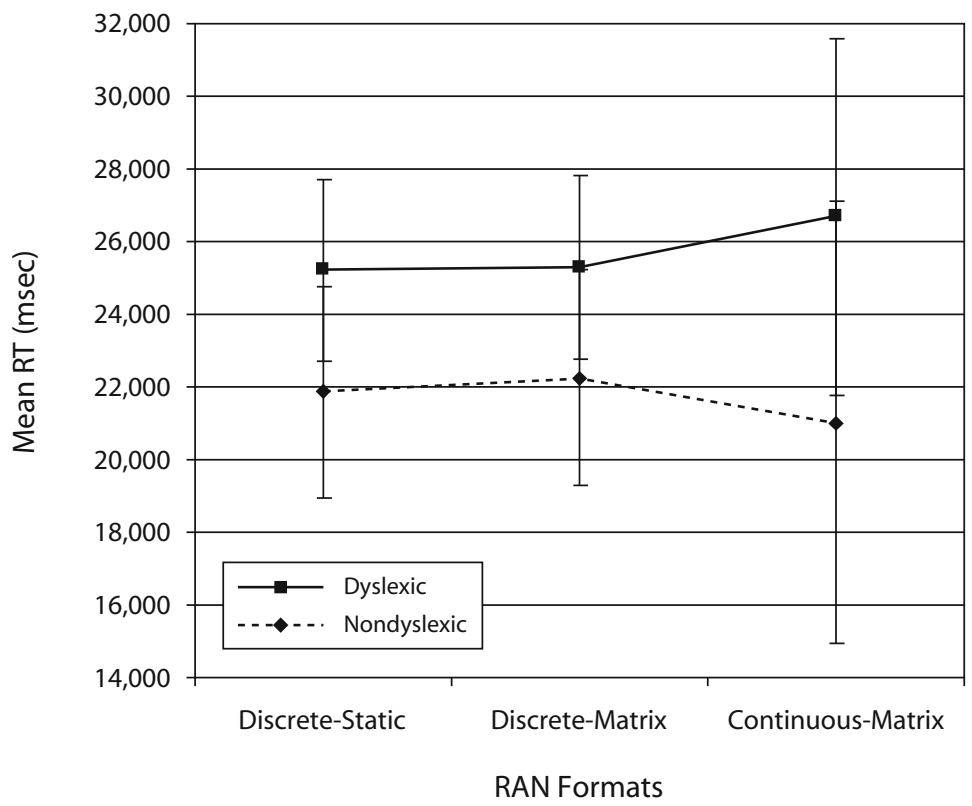

Figure 2. Dyslexic and nondyslexic performance on each presentation format (means and standard deviations). 
condition; indeed, they were marginally facilitated in this condition. In contrast, dyslexic readers were significantly slower to name items in the continuous-matrix condition when compared with nondyslexic readers' and their own performance in the discrete-static condition. We note here that, for both reading groups, performance on the novel discrete-matrix format did not differ statistically from the discrete-static format. We therefore found no evidence of impaired oculomotor control in this dyslexic group, in accordance with Hutzler et al. (2006), and we will not discuss this condition further.

The present findings shed light on the processes underlying RAN performance for both dyslexic and nondyslexic readers. Although some researchers have questioned the consistency with which discrete-naming tasks discriminate among reading groups (Walsh et al., 1988), our experiment demonstrated that discrete naming discriminates nondyslexic from dyslexic readers; furthermore, the naming-speed deficit is strong and pervasive even in a high-functioning (university student) sample of dyslexic readers. Our finding of significant differences in the discrete-static condition also suggests that rapid graphemic and/or phonological access of individual items is impaired in dyslexia, implying that one aspect of impaired RAN performance involves a deficit in rapid access to, or production of, single, highly familiar stimuli.

Of critical importance in this study was the differential effect of the continuous-matrix condition on readinggroup naming times. Previous studies have used regression analysis to show that continuous RAN formats are more consistent predictors of reading fluency than are discrete formats (e.g., Bowers \& Swanson, 1991), and that they reliably discriminate reading-group performance (e.g., Denckla \& Rudel, 1976). Our findings show that continuous lists of multi-item presentation dissociate nondyslexic versus dyslexic readers' naming speeds: As with studies involving object naming (e.g., Morgan $\&$ Meyer, 2005) and text reading (e.g., Sereno \& Rayner, 2000), nondyslexic readers' fluency was not impaired, and indeed showed a marginal advantage, when there was an opportunity to process more than one item in parallel or to exploit parafoveal information. Dyslexic readers also showed sensitivity to multiple items in RAN (consistent with Jones et al.'s, 2008, findings). In contrast to the effect on nondyslexic readers, however, multi-item processing impairs dyslexic readers' naming process, leading to slower naming times.

There are a number of possible explanations for dyslexic readers' impaired performance on the continuous RAN. First, domain-specific impairment in visual or phonological domains might result in competition between activated target and adjacent items' representations (Bouma, 1971; Clarke et al., 2005). Jones et al.'s (2008) findings, however, suggest that dyslexic readers' multi-item processing deficits occur across both visual and phonological domains and irrespective of whether the confusable item precedes or follows the target, suggesting domain-general impairment. Moreover, dyslexic readers' difficulty emerges when a verbal response is required. In the present study, dyslexics' impaired discrete and continuous RAN performance could reflect difficulty in selecting an item for production (see Jones et al., 2008; Ramus \& Szenkovits, 2008). In continuous versions of RAN, competition from a number of other visually present items would compound this deficit. Alternatively, simultaneous activation of multiple visual and phonological representations could delay the speed with which a grapheme can be bound to its corresponding phonological representation (see Breznitz, 2006).

\section{Conclusion}

This study aimed to identify the component processes of RAN that discriminate between dyslexic and nondyslexic readers' naming speeds. By extension, we sought to examine the low-level processes (graphemic, phonological, oculomotor, and sequencing) that are involved in reading fluency. Previous research has suggested that continuous lists (multi-item presentation of letter grids) are stronger predictors of reading fluency and are better able to discriminate among reading groups than are discrete (single-item presentation) lists (Bowers \& Swanson, 1991). Our findings clearly show why this is the case: The multi-item sequencing requirement of continuous RAN lists discriminates among reading groups because it benefits nondyslexic readers to some extent and significantly impairs dyslexic readers. Further work is now required to investigate whether a similar dissociation underlies dyslexic and nondyslexic differences on reading-fluency rates for connected text.

\section{AUTHOR NOTE}

We thank Martin Pickering, Martin Corley, Mateo Obregón, and Eddie Dubourg. Correspondence concerning this article should be addressed to M. W. Jones, Department of Psychology, University of Edinburgh, 7 George Square, Edinburgh EH8 9JZ, Scotland (e-mail: manon.wyn .jones@ed.ac.uk).

\section{REFERENCES}

BaAyen, R. H. (2008). Analyzing linguistic data: A practical introduction to statistics using $R$. Cambridge: Cambridge University Press.

Bates, D. M., Maechler, M., \& DaI, B. (2008). lme4: Linear mixedeffects models using S4 classes (R Package Version 0.999375-25) [Software]. Available from http://cran.r-project.org/web/packages/ lme4/index.html.

BisCaldi, M., GeZECK, S., \& STuhr, V. (1998). Poor saccadic control correlates with dyslexia. Neuropsychologia, 36, 1189-1202. doi:10.1016/ S0028-3932(97)00170-X

Bouma, H. (1971). Visual recognition of isolated lower-case letters. Vision Research, 11, 459-474.

Bowers, P. G., \& Swanson, L. B. (1991). Naming speed deficits in reading disability: Multiple measures of a singular process. Journal of Experimental Child Psychology, 51, 195-219. doi:10.1016/0022 -0965(91)90032-N

Breznitz, Z. (2006). Fluency in reading: Synchronization of processing. Mahwah, NJ: Erlbaum.

Castel, C., Pech-Georgel, C., George, F., \& Ziegler, J. C. (2008). Lien entre dénomination rapide et lecture chez les enfants dyslexiques [Link between rapid automatized naming and reading in dyslexic children]. Année Psychologique, 108, 395-421.

Chace, K. H., Rayner, K., \& Well, A. D. (2005). Eye movements and phonological parafoveal preview: Effects of reading skill. Canadian Journal of Experimental Psychology, 59, 209-217. doi:10.1037/ h0087476 
Clarke, P., Hulme, C., \& Snowling, M. (2005). Individual differences in RAN and reading: A response timing analysis. Journal of Research in Reading, 28, 73-86. doi:10.1111/j.1467-9817.2005.00255.x

Denckla, M. B., \& Cutting, L. E. (1999). History and significance of rapid automatized naming. Annals of Dyslexia, 49, 29-42. doi:10.1007/ s11881-999-0018-9

Denckla, M. B., \& Rudel, R. G. (1976). Rapid “automatized” naming (R.A.N.): Dyslexia differentiated from other learning disabilities. Neuropsychologia, 14, 471-479.

Georgiou, G. K., Parrila, R., \& Kirby, J. (2006). Rapid naming speed components and early reading acquisition. Scientific Studies of Reading, 10, 199-220. doi:10.1207/s1532799xssr1002_4

Hawelka, S., \& Wimmer, H. (2005). Impaired visual processing of multi-element arrays is associated with increased number of eye movements in dyslexic reading. Vision Research, 45, 855-863. doi:10.1016/j.visres.2004.10.007

HawelKa, S., \& Wimmer, H. (2008). Visual target detection is not impaired in dyslexic readers. Vision Research, 48, 850-852. doi:10.1016/j .visres.2007.11.003

Hutzler, F., Kronbichler, M., Jacobs, A. M., \& Wimmer, H. (2006). Perhaps correlational but not causal: No effect of dyslexic readers' magnocellular system on their eye movements during reading. Neuropsychologia, 44, 637-648. doi:10.1016/j .neuropsychologia.2005.06.006

Jones, M. W., Kelly, M. L., \& Corley, M. (2007). Adult dyslexic readers do not demonstrate regularity effects in sentence processing: Evidence from eye movements. Reading \& Writing, 20, 933-943. doi:10.1007/s11145-007-9060-3

Jones, M. W., Obregón, M., Kelly, M. L., \& Branigan, H. P. (2008) Elucidating the component processes involved in dyslexic and nondyslexic reading fluency: An eye-tracking study. Cognition, 109, 389-407.

Kooi, F. L., Toet, A., Tripathy, S. P., \& Levi, D. M. (1994). The effect of similarity and duration on spatial interaction in peripheral vision. Spatial Vision, 8, 255-279. doi:10.1163/156856894X00350

LERVÅ, A., \& Hulme, C. (in press). Rapid naming (RAN) taps a basic constraint on the development of early reading fluency. Psychological Science.

LEVI, D. M. (2008). Crowding-An essential bottleneck for object recognition: A mini-review. Vision Research, 48, 635-654. doi:10.1016/j visres.2007.12.009

Manis, F. R., Doi, L. M., \& Bhadha, B. (2000). Naming speed, phonological awareness, and orthographic knowledge in second graders. Journal of Learning Disabilities, 33, 325-333.

Manis, F. R., Seidenberg, M. S., Doi, L. M., McBride-Chang, C., \& Petersen, A. (1996). On the bases of two subtypes of developmental dyslexia. Cognition, 58, 157-195. doi:10.1016/0010 $-0277(95) 00679-6$

Morgan, J. L., \& Meyer, A. S. (2005). Processing of extrafoveal objects during multiple-object naming. Journal of Experimental Psychology: Learning, Memory, \& Cognition, 31, 428-442. doi:10.1037/0278 $-7393.31 .3 .428$

Pernet, C., Valdois, S., Celsis, P., \& Démonet, J.-F. (2006). Lateral masking, levels of processing and stimulus category: A comparative study between normal and dyslexic readers. Neuropsychologia, 44, 2374-2385. doi:10.1016/j.neuropsychologia.2006.05.003

Powell, D., Stainthorp, R., Stuart, M., Garwood, H., \& QuinLAN, P. (2007). An experimental comparison between rival theories of rapid automatized naming performance and its relationship to reading. Journal of Experimental Child Psychology, 98, 46-68. doi:10.1016/j .jecp. 2007.04 .003

Ramus, F., \& Szenkovits, G. (2008). What phonological deficit? Quarterly Journal of Experimental Psychology, 61, 129-141.
Savage, R. S., Frederickson, N., Goodwin, R., Patni, U., Smith, N., \& Tuersley, L. (2005). Relationships among rapid digit naming, phonological processing, motor automaticity, and speech perception in poor, average and good readers and spellers. Journal of Learning Disabilities, 38, 12-28.

Sereno, S. C., \& Rayner, K. (2000). Spelling-sound regularity effects on eye fixations in reading. Perception \& Psychophysics, 62, 402-409.

Shaywitz, S. E., \& Shaywitz, B. A. (2005). Dyslexia (specific reading disability). Biological Psychiatry, 57, 1301-1309. doi:10.1016/j .biopsych.2005.01.043

SNOWling, M. J. (2000). Dyslexia (2nd ed.). Oxford: Blackwell.

Sperling, A. J., Lu, Z.-L., Manis, F. R., \& Seidenberg, M. S. (2005). Deficits in perceptual noise exclusion in dyslexia. Nature Neuroscience, 8, 862-863.

TAroyan, N. A., Nicolson, R. I., \& FAwCETt, A. J. (2007). Behavioural and neurophysiological correlates of dyslexia in the continuous performance task. Clinical Neurophysiology, 118, 845-855. doi:10.1016/j .clinph.2006.11.273

Torgesen, J. K., Wagner, R. K., Rashotte, C. A., Burgess, S., \& Hecht, S. (1997). Contributions of phonological awareness and rapid automatic naming ability to the growth of word-reading skills in second- to fifth-grade children. Scientific Studies of Reading, 1, 161185. doi:10.1207/s1532799xssr0102_4

Wagner, R. K., Torgesen, J. K., Laughon, P., Simmons, K., \& Rashotte, C. A. (1993). Development of young readers' phonological processing abilities. Journal of Educational Psychology, 85, 83103. doi:10.1037/0022-0663.85.1.83

Walsh, D. J., Price, G. G., \& Gillingham, M. G. (1988). The critical but transitory importance of letter naming. Reading Research Quarterly, 23, 108-122. doi:10.2307/747907

Wilkinson, G. S. (1993). The Wide Range Achievement Test: Administration manual. Wilmington, DE: Wide Range.

Wimmer, H., MAYringer, H., \& LANDERL, K. (2000). The double-deficit hypothesis and difficulties in learning to read a regular orthography. Journal of Educational Psychology, 92, 668-680. doi:10.1037/0022 $-0663.92 .4 .668$

Wolf, M., \& Bowers, P. G. (1999). The double-deficit hypothesis for the developmental dyslexias. Journal of Educational Psychology, 91, 415-438. doi:10.1037/0022-0663.91.3.415

Wolf, M., \& OBregón, M. (1992). Early naming deficits, developmental dyslexia, and a specific deficit hypothesis. Brain \& Language, 42, 219-247. doi:10.1016/0093-934X(92)90099-Z

Young, A., \& Bowers, P. G. (1995). Individual difference and text difficulty determinants of reading fluency and expressiveness. Journal of Experimental Child Psychology, 60, 428-454. doi:10.1006/ jecp. 1995.1048

\section{NOTES}

1. This information is based on a recent PsycINFO database search.

2. We did not obtain IQ measures for the reading groups. Potential group differences in IQ were minimized, however, by the fact that all participants came from the same, high-functioning, university undergraduate population.

3. A sum of RTs across each trial is the only viable means of data analysis because of the nature of the continuous-list format and the requirement that data between the continuous lists and discrete trials be compatible for analysis; see Denckla and Cutting (1999, pp. 31-32).

(Manuscript received October 30, 2008, revision accepted for publication February 14, 2009.) 